\title{
Fc-receptor-mediated phagocytosis is regulated by mechanical properties of the target
}

\author{
Karen A. Beningo and Yu-li Wang* \\ University of Massachusetts Medical School, Department of Physiology, 377 Plantation, Room 327, Worcester, MA 01605, USA \\ *Author for correspondence (e-mail: yuli.wang@umassmed.edu) \\ Accepted 13 November 2001 \\ Journal of Cell Science 115, 849-856 (2002) (C) The Company of Biologists Ltd
}

\section{Summary}

Phagocytosis is an actin-based process used by macrophages to clear particles greater than $0.5 \mu \mathrm{m}$ in diameter. In addition to its role in immunological responses, phagocytosis is also necessary for tissue remodeling and repair. To prevent catastrophic autoimmune reactions, phagocytosis must be tightly regulated. It is commonly assumed that the recognition/ selection of phagocytic targets is based solely upon receptor-ligand binding. Here we report an important new criterion, that mechanical parameters of the target can dramatically affect the efficiency of phagocytosis. When presented with particles of identical chemical properties but different rigidity, macrophages showed a strong

preference to engulf rigid objects. Furthermore, phagocytosis of soft particles can be stimulated with the microinjection of constitutively active Rac1 but not RhoA, and with lysophosphatidic acid, an agent known to activate the small GTP-binding proteins of the Rho family. These data suggest a Rac1-dependent mechanosensory mechanism for phagocytosis, which probably plays an important role in a number of physiological and pathological processes from embryonic development to autoimmune diseases.

Key words: Mechanical sensing, Cytoskeleton, GTPase, Rac1, Lysophosphatidic acid, Phosphotyrosine

\section{Introduction}

Phagocytosis is mediated by a number of mechanisms, among which Fc-receptor-mediated phagocytosis is the most extensively studied (Greenberg and Silverstein, 1993; Metchnikoff, 1968; Aderem and Underhill, 1999). Despite what is already known of this mechanism, large gaps remain in our knowledge of how the process is initiated, particularly how a specific target is selected for phagocytosis. An essential component for Fc-mediated phagocytosis is the Fc receptor family, which binds to antibody-coated objects and is primarily responsible for the initial recognition (Greenberg and Silverstein, 1993; Metchnikoff, 1968; Aderem and Underhill, 1999). The binding of ligands causes clustering of Fc-receptors and activation of a number of signaling events including tyrosine phosphorylation (Aderem and Underhill, 1999; Greenberg et al., 1993), which eventually lead to the recruitment of actin and actin-associated proteins to form a phagocytic cup (Greenberg and Silverstein, 1993; Metchnikoff, 1968; Aderem and Underhill, 1999; May and Machesky, 2001).

Many aspects of Fc-receptor-mediated phagocytosis resemble events at the leading edge of a migrating cell. Both processes are actin-mediated and both involve adhesion, receptor clustering, tyrosine phosphorylation, lamellipodial/ pseudopodial extension, and force generation (Aderem and Underhill, 1999; Sheetz et al., 1998). For cell migration, it is becoming increasingly clear that both chemical and physical parameters of the substrate play an important role (Sheetz et al., 1998). For example, transient mechanical stimuli can activate cell migration and neurite growth (Verkhovsky et al., 1999; Bray, 1984), possibly through the reorganization of the actin cytoskeleton and focal adhesions (Heidemann et al., 1999; Riveline et al., 2001). In addition, substrate flexibility was found to affect both the rate and direction of fibroblast migration (Pelham and Wang, 1997; Lo et al., 2000). The similarities between lamellipodia extending on a substrate and phagocytic cups forming around the target raises the possibility that mechanical signaling may play an equally important role in phagocytosis.

To test this hypothesis, we have fed macrophages with polyacrylamide microbeads of identical surfaces but different stiffness. Our results provide convincing evidence that target rigidity indeed plays a determining role in phagocytosis. We show that adhesion, cortical cytoskeleton, and protein phosphorylation respond differently to rigidity signals and that the mechanosensitivity is regulated by lysophosphatidic acid (LPA) and the small GTP-binding protein Rac1.

\section{Materials and Methods \\ Preparation of polyacrylamide beads and sheets}

To prepare polyacrylamide beads, a microemulsion was created by dissolving $10.2 \mathrm{mg}$ Bis (2-ethylhexyl) sulfosuccinate (AOT; Fluka, Milwaukee, WI) in $1 \mathrm{ml}$ of toluene (Kunioka and Ando, 1996; Candau and Leong, 1985), followed by the addition of $100 \mu$ of an activated aqueous solution containing $8 \%$ acrylamide and $0.05 \%$ or $0.2 \%$ bisacrylamide, $20 \mathrm{mg} / \mathrm{ml}$ FITC-dextran (464 kDa; Molecular Probes, Eugene, OR) and $0.2 \%$ acrylic acid, while stirring under a nitrogen stream. The beads were allowed to polymerize for 1 hour with constant stirring under nitrogen, then recovered by centrifugation at $23 \mathrm{~g}$ for 5 minutes and washed repeatedly in methanol. Following multiple washes in phosphate buffered saline (PBS) the beads were stored at $4^{\circ} \mathrm{C}$. Polyacrylamide sheets were prepared as previously 
described (Wang and Pelham, 1998), and the Young's modulus was determined as described (Lo et al., 2000).

Bovine serum albumin (BSA) was covalently coupled to the polyacrylamide beads or sheets after activating the surface with 1ethyl-3-(3-dimethylaminopropyl) (EDC; Pierce, Rockford, IL), followed by incubation with affinity purified rabbit anti-BSA IgG (Cappel, Aurora, $\mathrm{OH}$ or Sigma, St Louis, MO) for 1 hour at $24^{\circ} \mathrm{C}$. Large particles $(>6 \mu \mathrm{m})$ were removed by centrifugation in a microfuge at $300 \mathrm{~g}$ for 3 minutes. The remaining beads, $1-6 \mu \mathrm{m}$ in diameter, were used for the experiments. In some experiments, beads were further incubated with $3 \mu \mathrm{M}$ LPA (Avanti Polar Lipids, Alabaster, AL) in PBS for 1 hour at $24^{\circ} \mathrm{C}$. The concentration of beads was determined before each phagocytosis experiment as follows: a 30 $\mu \mathrm{l}$ sample of opsonized beads was placed onto a slide, dispersed uniformly under a $22 \mathrm{~mm}$ circular coverslip, and allowed to settle for at least 30 minutes before counting. The concentration was calculated based on the average number of beads counted within five square areas of $200 \times 200$ pixels.

To determine the extent of antibody coating, opsonized beads were labeled with fluorescent secondary antibodies for 1 hour at $24^{\circ} \mathrm{C}$ (Alexa 546 anti-rabbit IgG, Molecular Probes, Eugene, OR), and the fluorescence intensity of single beads measured with a cooled CCD camera coupled to a microscope (Fig. 1a). In some experiments, the results of microscopy photometry were verified with flow cytometry. The density of surface coating on sheets was tested as previously described (Fig. 1b,c) (Lo et al., 2000), using $1 \mu \mathrm{m}$ Fluorsbrite carboxylate beads coated with antibodies against rabbit IgG (Polysciences, Warrington, PA).

\section{Preparation of macrophages and induction of phagocytosis}

Bone-marrow-derived macrophages from 4-6-week-old $\mathrm{C} 3 \mathrm{H} / \mathrm{HeJ}$ mice were prepared essentially as described previously (Swanson, 1989), using recombinant huCSF-1 (R\&D Systems, Minneapolis, $\mathrm{MN})$ to induce the differentiation. Equal numbers $\left(\sim 1 \times 10^{6}\right.$ cells per $35 \mathrm{~mm}$ diameter area) of 5-8 day old cells, in growth medium without CSF-1, were plated onto coverslips 24 hours before the experiments. The cells were rinsed and acclimated for 10 minutes at $37^{\circ} \mathrm{C}$ in PBS. Equal numbers of beads $\left(\sim 1 \times 10^{7}\right.$ beads per $35 \mathrm{~mm}$ diameter area) were then presented to the cells and incubated at $37^{\circ} \mathrm{C}$ for 45 minutes for the uptake assays, or 10 minutes for immunofluorescence staining. Cells were then fixed as previously described (Pelham and Wang, 1997), and the ingested beads counted with a combination of phase and fluorescence optics. Completely engulfed beads appeared as bright green particles in fluorescence optics (Fig. 2b), but were phasedense and barely detectable in phase optics (Fig. 2a; Fig. 3c). This relationship between phase morphology and engulfment was confirmed with $3 \mathrm{D}$ reconstruction of optical sections of cells stained with fluorescent phalloidin (Fig. 2c). The phagocytic index is defined as the average number of beads engulfed per cell after 45 minutes of incubation.

For the experiments of frustrated phagocytosis, cells in PBS were allowed to interact with opsonized polyacrylamide sheets for 45 minutes before fixation and staining with rhodamine phalloidin (Molecular Probes, Eugene, $\mathrm{OH}$ ). Those with lamella extending in a radially symmetric fashion were counted as positive (Fig. 3e).

\section{Fluorescent staining, microinjection and microscopy}

Fixed cells were blocked with $1 \% \mathrm{BSA}$ and stained for 1 hour at $37^{\circ} \mathrm{C}$ with rhodamine phalloidin for filamentous actin (Molecular Probes), with a monoclonal antibody pTyr-clone $4 \mathrm{G} 10$ for phosphotyrosine (1:100; Upstate Biotechnology, Lake Placid, NY), or with a monoclonal antibody clone 349 for paxillin (1:200; ICN, Costa Mesa, CA). Anti-mouse IgG coupled to the Alexa 546 fluorophore (Molecular Probes) was used at 1:1000 dilution as the secondary antibody.
Constitutively active L61Rac1 and L63RhoA, and dominant negative N17Rac1-GST were purchased from Cytoskeleton (Denver, $\mathrm{CO}$ ), and resuspended and stored as recommended by the manufacturer. C3 transferase was received as a gift from $\mathrm{S}$. Narumiya (Kyoto University, Kyoto, Japan) and used as described previously (O'Connell et al., 1999). The protein or carrier solution was microinjected as previously described (Wang, 1994), into murine-derived macrophages starved of m-CSF for 24 hours or into REF cells serum starved for 16 hours as controls. Rac 1 and RhoA proteins were injected at a needle concentration of $800 \mathrm{ug} / \mathrm{ml}$, and $\mathrm{C} 3$ was injected at a needle concentration of $200 \mathrm{ug} / \mathrm{ml}$. The carrier solution contained $7 \mathrm{mg} / \mathrm{ml}$ rhodamine dextran $(70 \mathrm{kDa}$, lysine fixable; Molecular Probes) as a marker. Following microinjection the cells were allowed to recover for 20 minutes in $37^{\circ} \mathrm{C}$ PBS and the phagocytosis experiments were carried out as described above. The number of beads ingested by injected cells was counted after fixation.

Cells were observed with a Zeiss Axiovert 10 or Axiovert TV100 microscope, equipped with a quartz halogen lamp and a 40×, NA 0.75 Plan-Neofluar objective lens. Images were recorded with a slow-scan cooled CCD camera (ST133 with CCD57 chip; Roper Scientific, Trenton, NJ) and processed for background subtraction. Optical sections of phalloidin images were processed with a custom feature detection program that highlights linear structures and outlines of phagosomes, and reconstructed into perspective views for stereo visualization.
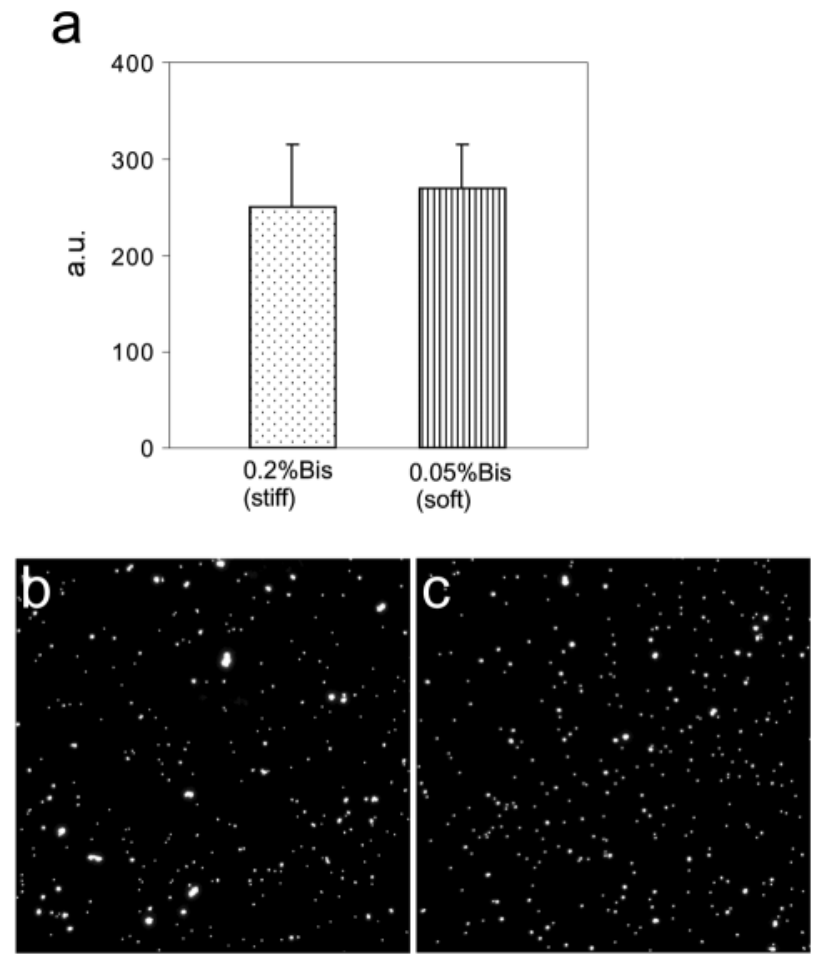

Fig. 1. Similar opsonization of hard and soft polyacrylamide microbeads and sheets. (a) Average fluorescent intensities of opsonized beads labeled with red fluorescent secondary antibodies. Intensities were measured with 100 each of stiff and soft beads from three preparations. Error bars indicate the standard deviation between each experiment. (b,c) Images of stiff (b) or soft (c) polyacrylamide surfaces coated with anti-BSA IgG and labeled with fluorescent carboxylate beads coated with anti-rabbit antibodies. The average intensities were within $7 \%$ of each other for the beads and $15 \%$ for the sheets. 

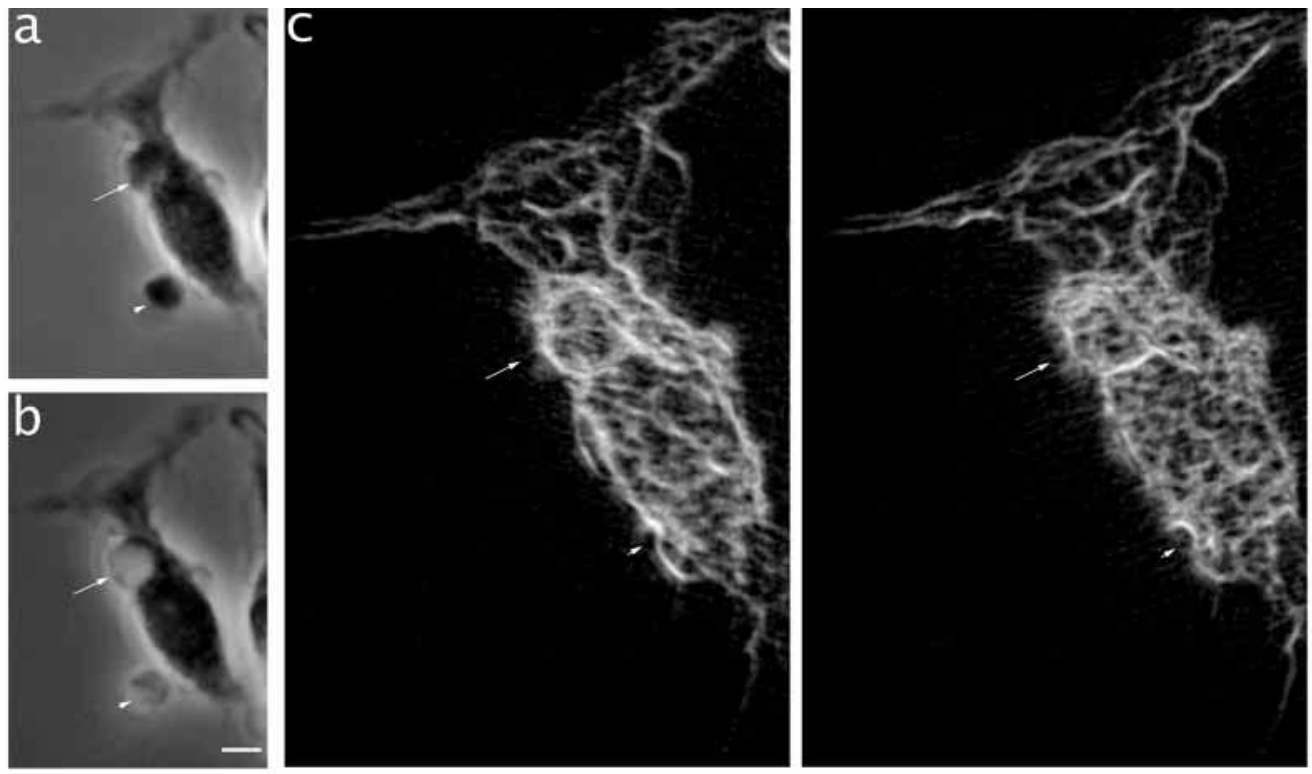

Fig. 2. Detection of ingested polyacrylamide beads.

Macrophages were fed with stiff, opsonized polyacrylamide beads with fluorescent dextran trapped inside. Unlike beads that stuck to the side of the cell (arrowhead), internalized beads are not readily visible with phase optics (a; long arrow). However, a combination of phase and fluorescence optics easily reveals all the beads (b; arrow and arrowheads). (c) Stereo reconstruction of the same cell stained with rhodamine phalloidin, showing a closed phagocytic cup around the internalized bead (long arrow). A patch of actin is concentrated beneath the external bead (arrowhead). Bar, $4 \mu \mathrm{m}$.

\section{Results}

\section{Phagocytosis is a mechanosensitive process}

To address the role of mechanical signaling in Fc-receptor mediated phagocytosis, we prepared polyacrylamide microbeads of variable rigidity. The stiffness of the beads was controlled, without changing the chemical properties of the surface, by varying the concentration of bis-acrylamide crosslinker while maintaining a constant total acrylamide concentration. Measurements of Young's modulus with corresponding polyacrylamide sheets indicated a greater than threefold difference in flexibility between the beads prepared with $0.2 \%$ bis-acrylamide (stiff beads) and those with $0.05 \%$ bis-acrylamide (soft beads). Microbeads were covalently coupled with BSA and opsonized with rabbit anti-BSA IgG (see Materials and Methods). Measurements of the fluorescence intensity after labeling beads with fluorescent secondary antibody indicated that hard and soft beads were coated with a similar density of IgG (Fig. 1a).

As shown in Fig. 3a, macrophages showed a sixfold preference for the stiff over the soft opsonized beads. Naked beads or beads coated with BSA alone served as controls; both of which showed poor adhesion to the cell surface and a very low phagocytic index $(<0.05)$. Although opsonized soft particles were ingested poorly, they were able to adhere to the surface of macrophages (Fig. 3f,g). In addition, no increase in the number of ingested soft beads was observed after an extended incubation of 2 hours, indicating that the difference between stiff and soft beads was not due to different rate of ingestion. Instead, the observations suggest that phagocytosis was inhibited downstream of receptor binding.

In a parallel experiment, we plated macrophages on polyacrylamide sheets of different rigidity but opsonized with a similar density of IgG (Fig. 1b,c). The cells spread dramatically in an attempt to engulf the substrate (North, 1968), a phenomenon termed frustrated phagocytosis (Henson, 1971; Rabinovitch and DeStefano, 1973) (Fig. 3e). The results again revealed a strong preference for the stiff over the soft substrate (Fig. 3b,e,h).

To determine whether the binding of stiff or soft beads caused a global positive or negative response of phagocytosis, macrophages were incubated with a mixture of equal numbers of opsonized hard and soft beads, labeled with different colors of fluorescent dextran. Comparisons of phagocytic indices of hard versus soft beads showed no difference in the phagocytic uptake of either the hard or soft beads when presented in combination or individually, suggesting that target stiffness elicits local responses that determine the efficiency of phagocytosis.

\section{Target rigidity affects the organization of actin filaments but not paxillin or phosphotyrosine}

Both actin cytoskeleton and adhesion structures are involved in phagocytosis (Aderem and Underhill, 1999; May and Machesky, 2001) and both are known to respond to mechanical stimuli in fibroblasts (Heidemann et al., 1999; Riveline et al., 2001). Staining with rhodamine phalloidin showed that actin filaments were concentrated beneath $85 \%$ of the stiff beads $(n=100)$, but only $11 \%$ of the soft beads $(n=100$; Fig. 4a,b). This lack of actin organization is probably responsible for the failure of cells to engulf soft particles. However, staining of paxillin (Fig. 4e,f) or vinculin (data not shown), two proteins localized to the adhesive sites of $\mathrm{Fc}$-mediated phagocytic cups (Greenberg et al., 1994; Allen and Aderem, 1996), showed that qualitatively both proteins became localized beneath stiff or soft beads. Thus the initial recruitment of these proteins to nascent phagocytic cups probably occurs upstream of actin assembly, independent of the stiffness of the target.

It was previously reported that protein tyrosine phosphorylation increases during both phagocytosis (Greenberg et al., 1993) and mechanical stimulations (Pelham and Wang, 1997; Schmidt et al., 1998). This response may play a role in mediating actin organization upon the binding of stiff beads. However, both stiff $(99 \% ; n=100)$ and soft beads (98\%; 

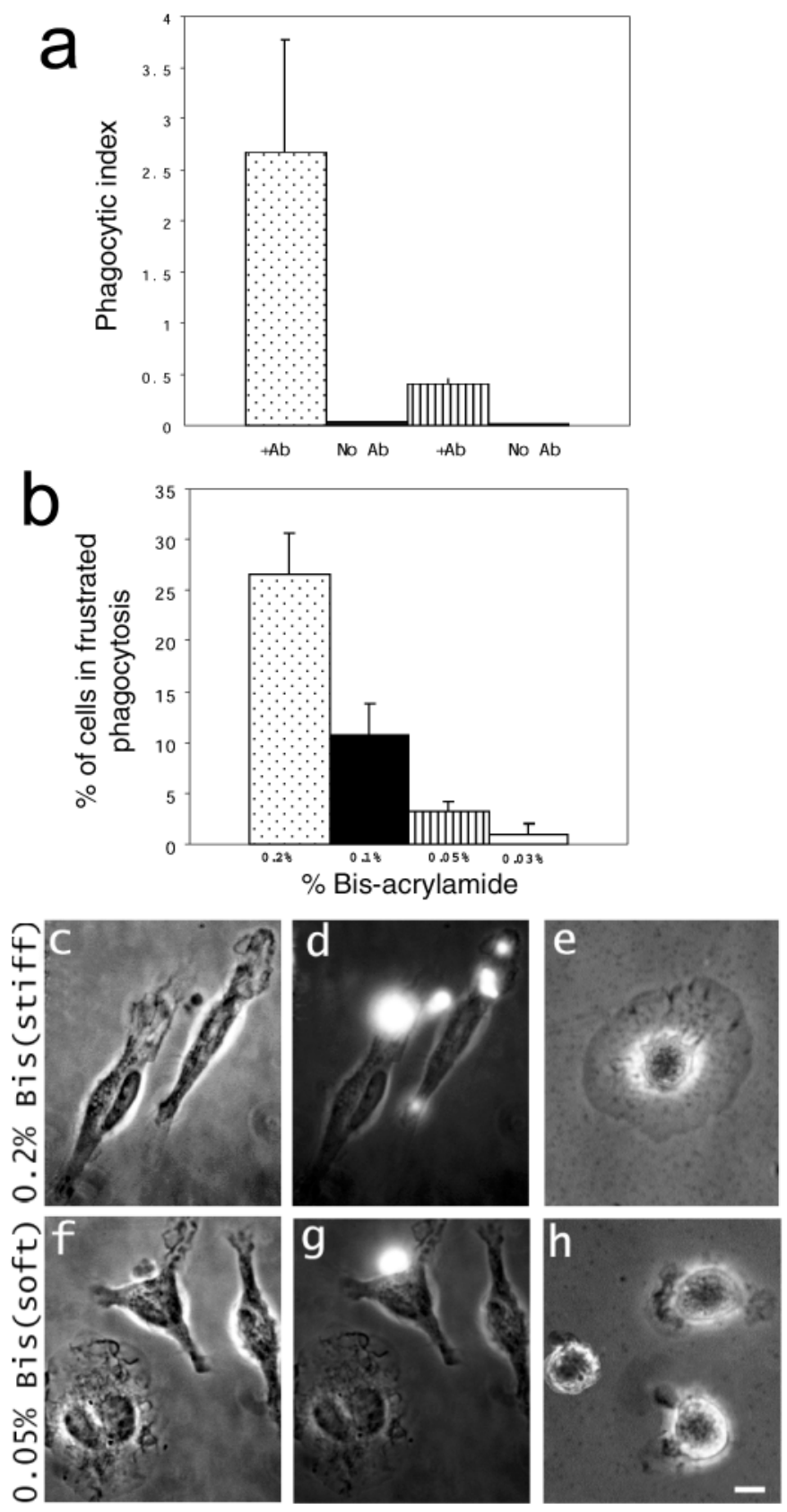

Fig. 3. Efficiency of phagocytosis of stiff or soft objects during Fcreceptor-mediated phagocytosis. (a) Stiff polyacrylamide beads (left two bars) are six times more likely to be phagocytosed than soft beads (right two bars). Beads without antibody coating are poorly phagocytosed. Phagocytic index is defined as the average number of beads internalized per cell. These data were compiled from six independent experiments with 100 cells counted in each experiment. (b) Frustrated phagocytosis on progressively softer opsonized polyacrylamide sheets. Data were compiled from three independent experiments. Stiff sheets cause sixfold more cells to undergo frustrated phagocytosis than do soft sheets. Error bars indicate the standard deviation between experiments. Panels c-e show images of macrophages phagocytosing opsonized stiff beads (c-d) and sheets (e). Internalized beads can be easily discriminated from beads that are merely attached to the cell surface based on fluorescence and phase images. Panels f-h show cells that failed to phagocytose opsonized soft beads (f-g) or sheets (h). Bar, $10 \mu \mathrm{m}$.
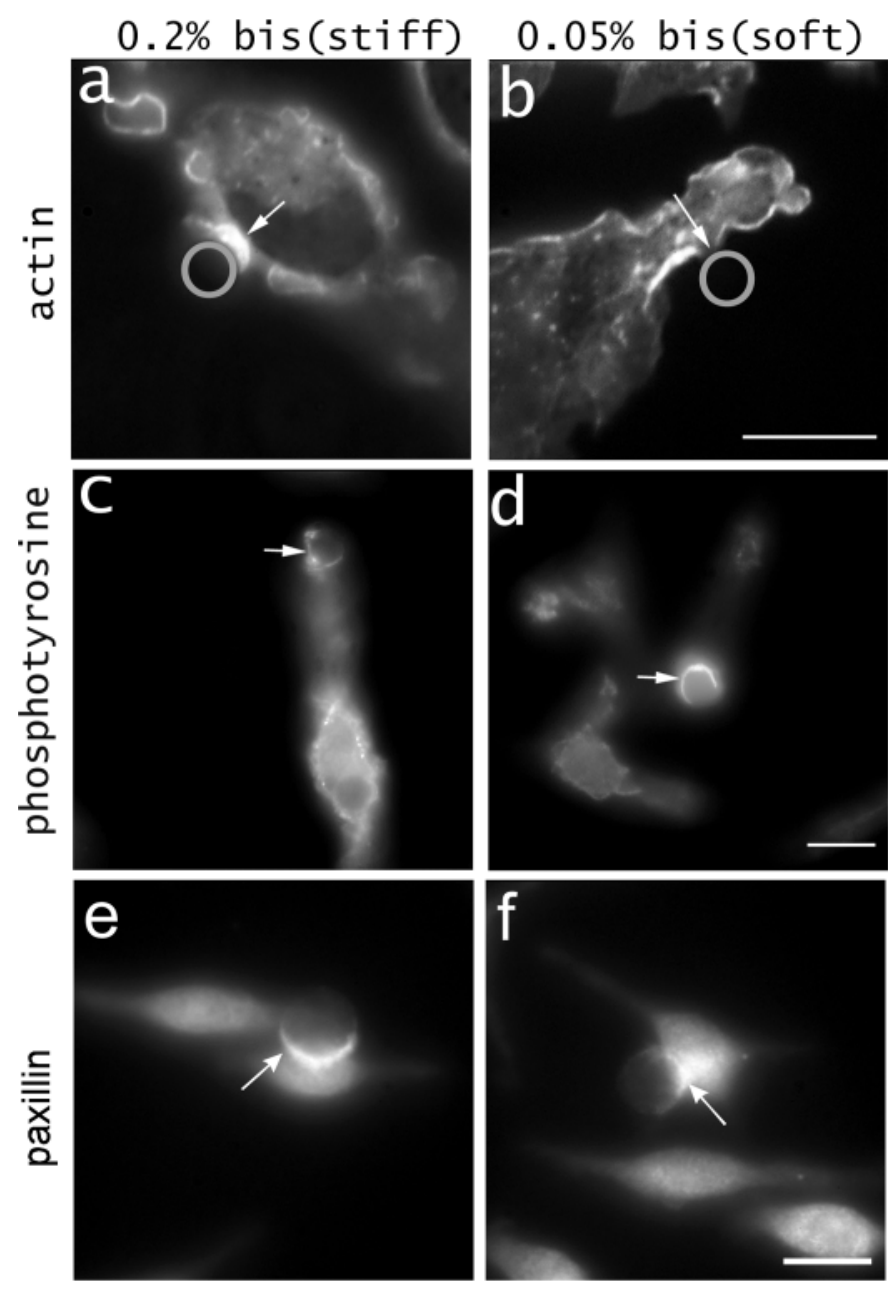

Fig. 4. Organization of actin filaments, paxillin and phosphotyrosine following the binding of polyacrylamide beads. Actin (a),

phosphotyrosine (c), and paxillin (e) are concentrated beneath bound stiff beads (arrows). However, only phosphotyrosine (d) and paxillin (f), but not actin (b), are concentrated beneath bound soft beads

(b,d,f; arrows). Beads are represented as gray circles in panels a and b. Owing to the high intensity of actin staining, weak fluorescence of these beads is not detectable in these prints. Bar, $6 \mu \mathrm{m}$.

$n=100)$ induced a concentration of phosphotyrosine at the binding site (Fig. 4c,d), suggesting that tyrosine phosphorylation also lies upstream of the mechanosensitive mechanism. However, it is possible that stiff beads may induce quantitatively a higher extent of phosphorylation at steady state. It is also possible that mechanosensing may involve differential tyrosine phosphorylation of a minor protein, which cannot be discerned by the global staining.

\section{The small GTPase Rac1 is involved in mechanosensing} The small GTPases are effectors of cytoskeletal organization and are known to be involved in phagocytosis (May and Machesky, 2001). To test the involvement of RhoA and Rac1 in mechanosensing, we microinjected constitutively active L63RhoA and L61Rac1 into macrophages prior to the presentation of hard or soft beads. Interestingly, constitutively 

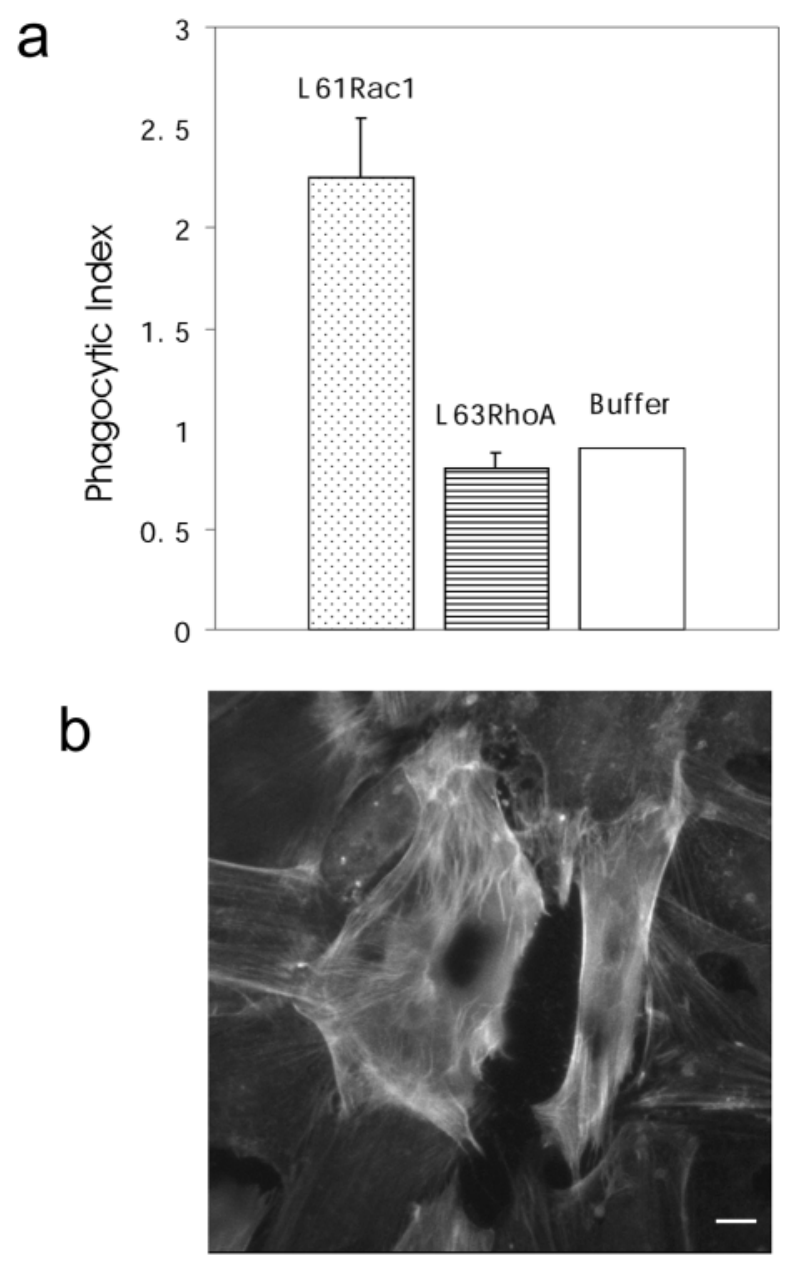

Fig. 5. Effects of constitutively active Rho GTPases on the phagocytosis of soft particles. (a) Microinjection of L61Rac1, but not L63RhoA, stimulates the uptake of soft beads. Protein solutions were microinjected into 230 (L61Rac1), 120 (L63RhoA) or 100 cells (buffer control), before the presentation of opsonized soft beads. (b) Rhodamine phalloidin staining of serum-starved REF cells microinjected with L63RhoA reveals strong staining of stress fibers compared with surrounding uninjected cells, confirming the activity of the injected protein. Error bars indicate the standard deviation between three independent experiments. Bar, $10 \mu \mathrm{m}$.

active Rac1 not only created extensive ruffling and lamellipodial activities as previously reported (Allen et al., 1997), but also stimulated the ingestion of opsonized soft beads to a level similar to that for stiff beads (Fig. 5a). Moreover, microinjection of dominant negative N17Rac1 inhibited the uptake of hard particles (Fig. 7a), consistent with previous reports (Caron and Hall, 1998).

By contrast, microinjection of L63RhoA had no effect on the ingestion of stiff or soft beads (Fig. 5a). Similarly, microinjection of the $\mathrm{C} 3$ transferase, a specific inhibitor of Rho, had no effect on phagocytosis (Fig. 7b), although it did cause the cells to spread out as described in Bac1.2F5 cells (Allen et al., 1997). Serum-starved REF cells did respond to L63RhoA by forming stress fibers (Fig. 5b) (Ridley and Hall, 1992), and to C3 transferase by rounding up (Fig. 7c) confirming the activity of these proteins. Together, these
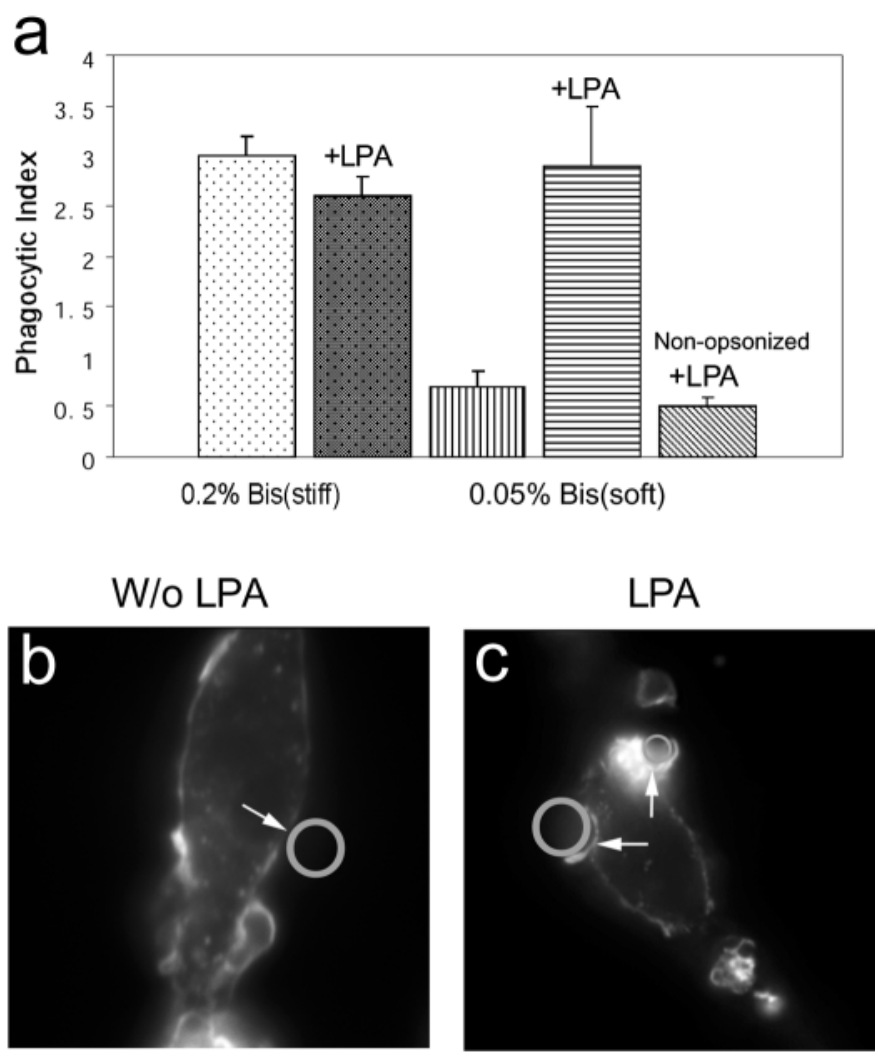

Fig. 6. Stimulation of phagocytosis of soft particles by LPA. (a) Opsonized beads were incubated with $3 \mu \mathrm{M}$ LPA before presenting to murine macrophages and the phagocytic index determined as in Fig. 1. The data were compiled from four independent experiments, error bars indicate the standard deviation between experiments. LPA-stimulated the ingestion of opsonized soft beads, but not soft beads labeled with BSA alone. LPA also induced a concentration of actin filaments beneath soft beads (c, arrows), when compared with the control (b, arrows). Bar, $3 \mu \mathrm{m}$.

results support the notion that Rac1, but not RhoA, plays an important role in mechanosensing during Fc-receptor-mediated phagocytosis.

\section{LPA stimulates phagocytosis of soft particles}

To attempt to identify external factors that may modulate mechanosensing of phagocytosis, we incubated opsonized beads with lysophosphatidic acid (LPA), a natural serum component that functions as an activator of several signaling pathways including the Rho GTPases (Ridley and Hall, 1992; Moolenaar 1999; Koh et al., 1998). LPA greatly stimulated the phagocytosis of opsonized soft beads, to an extent similar to that of stiff particles (Fig. 6a). Actin filaments became concentrated beneath $85 \%$ of the soft beads ( $n=200$; Fig. $6 \mathrm{~b}, \mathrm{c}$ ), similar to what was found with stiff beads. However, incubation of stiff beads with LPA did not induce further enhancement of phagocytosis, nor did cells engulf nonopsonized soft beads coated with BSA and LPA (Fig. 6a). Thus, rather than activating an independent, indiscriminate mechanism of phagocytosis, LPA probably elicits a signal normally stimulated by the rigidity of the opsonized target. 

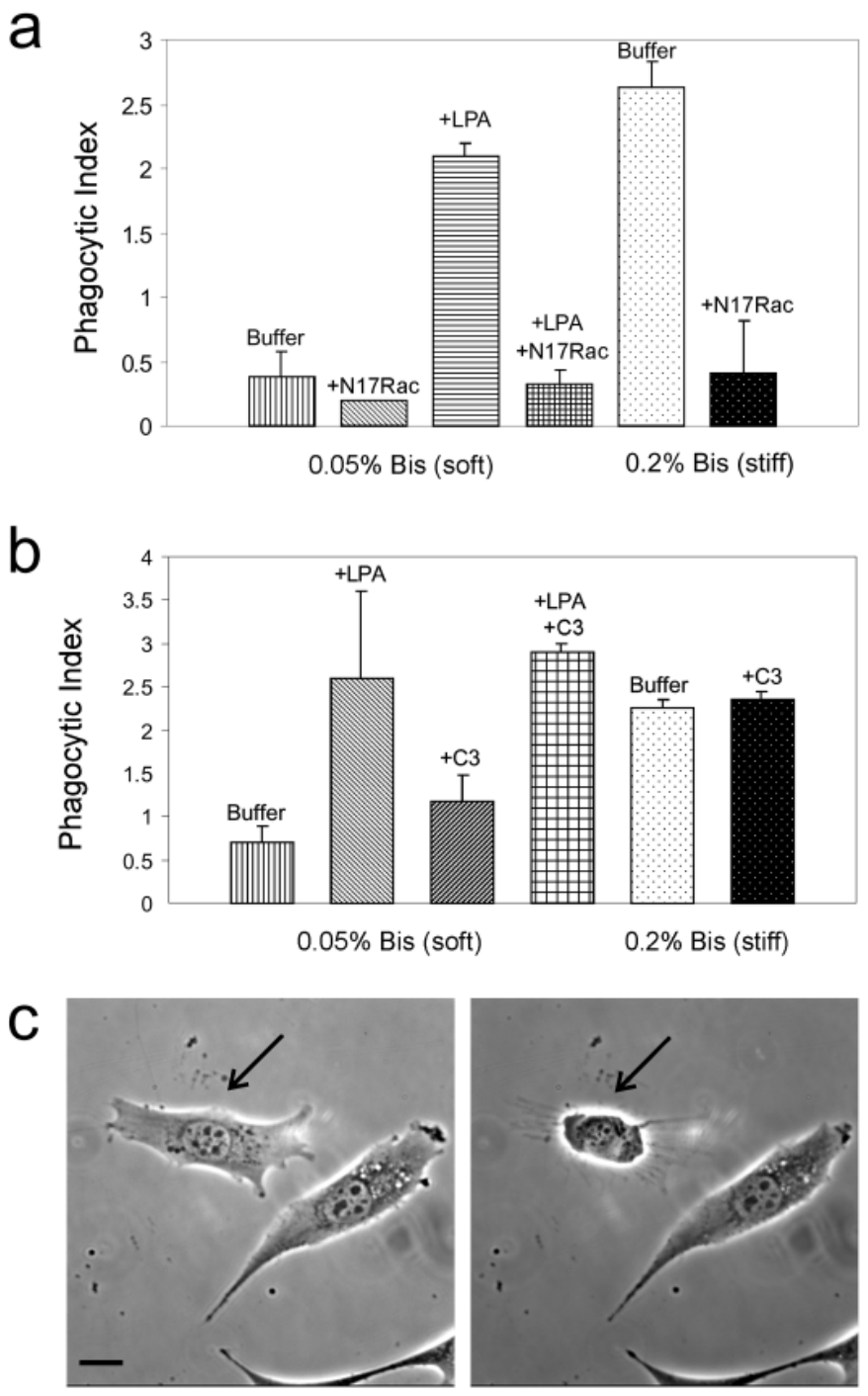

Fig. 7. Inhibition of LPA-stimulated phagocytosis of soft particles by dominant negative Rac1. (a) Macrophages microinjected with N17Rac1 or buffer were fed with opsonized hard or soft beads, with or without the additional incubation with LPA. The phagocytic index for each condition was determined with a total of 70-100 cells in three independent experiments. (b) Macrophages microinjected with C3 transferase or buffer were fed with opsonized beads, with or without the additional incubation with LPA. The phagocytic index for each condition was determined with a total of 120-150 cells in three independent experiments. Error bars indicate the standard deviation between experiments. Efficacy of the $\mathrm{C} 3$ transferase was confirmed by the induction of cell retraction upon microinjection into serum-starved REF-52 cells. Bar, $10 \mu \mathrm{m}$.

It is possible that LPA stimulates phagocytosis of soft particles through the activation of Rac1, as was suggested in some systems (Moolenaar, 1999). Consistent with this idea, dominant negative Rac1 abolished the ingestion of LPA-coated soft particles (Fig. 7a), while C3 had no effect (Fig. 7b). However, we detected no global elevation of Rac1 activities following the treatment with LPA alone (data not shown), suggesting that the stimulation of Rac1 by LPA requires Fc binding and thus may be localized near phagocytic sites.

\section{Discussion}

Previous studies have indicated that phagocytosis is affected by several physical parameters of the target. The size of a particle can dictate the efficiency of uptake and the extent of actin organization (Cannon and Swanson, 1992; Koval et al., 1998), while the shape of the target may also affect the ingestion process (Donaldson et al., 1992). The present results indicate that macrophages use rigidity and/or force input from a surface-bound particle as part of the cues to determine whether the target should be ingested. This intriguing process adds a novel selection criterion for phagocytosis. Although the detailed mechanism remains to be defined, we have obtained significant clues from the differential responses of proteins associated with phagosomes.

Our results suggest that, at least at a qualitative level, the engagement of $\mathrm{Fc}$ receptors with $\mathrm{IgG}$ is sufficient for activating tyrosine phosphorylation and the reorganization of paxillin and vinculin at the binding site. However soft beads were unable to stimulate the assembly of actin filaments required for the formation and closure of phagosomes. This mechanosensing mechanism probably involves a force-dependent probing step, which lies downstream of the initial steps of ligand binding and tyrosine phosphorylation. Owing to the difficulty in measuring phagosome-associated proteins, we cannot rule out the possibility that stiff targets stimulate a further increase in tyrosine phosphorylation and vinculin/paxillin organization through a feedback mechanism, as was observed at focal adhesions of fibroblasts (Pelham and Wang, 1997).

Previous studies have implicated the Rho-family GTPases in regulating cortical actin reorganization during $\mathrm{Fc}$-mediated phagocytosis (Chimini and Chavier, 2000). The effects of this family of proteins, including Rho, Rac and $\mathrm{Cdc} 42$, on the actin cytoskeleton are well established in non-macrophage cells (Nobes and Hall, 1999; Chrzanowska-Wodnicka and Burridge, 1996; Amano et al., 1996). Although these proteins have also been localized to phagocytic cups (Caron and Hall, 1998), their exact role in phagocytosis is less clear. Experiments with C3 transferase have yielded contradictory results as to the involvement of RhoA in Fc-mediated phagocytosis, while other studies have indicated the involvement of Rac1 in mediating pseudopod extension and particle engulfment (Caron and Hall, 1998; Hackam et al., 1997; Massol et al., 1998; Cox et al., 1997; Castellano et al., 2000). Our results support the notion that Rac1, but not RhoA, is involved in Fcreceptor mediated phagocytosis. In addition, we provide evidence that Rac1 mediates a mechanosensing process that leads to the preferential ingestion of stiff particles. In the simplest scenario, mechanical input from rigid particles causes the activation of Rac1, which in turn activates the assembly of actin filaments at phagocytic cups. There are three known pathways, involving the PAK/LIM kinases, phosphoinositides, and the Arp2/3 complexes respectively, through which activated Rac1 could induce actin polymerization. Components of all three pathways have been localized to the phagocytic cup (Dharmawardhane et al., 1999; May et al., 2000; Botelho et al., 2000).

Our observation that LPA bypasses the mechanosensing mechanism is intriguing, given the stimulation of Rho by LPA in many other systems and the lack of involvement of RhoA in the present experiments. However, LPA may activate alternative GTP binding proteins during phagocytosis. In the 
present study, the effects of LPA were inhibited by dominant negative Rac1, suggesting that Rac1, instead of RhoA, is the primary effector of the LPA. This LPA-modulated mechanosensing mechanism is likely to have broad implications in vivo. For example, bacteria, yeast, and other pathogens with cell walls are usually more rigid than the surrounding tissue they invade (Doyle and Marquis, 1994; Thwaites and Mendelson, 1991), thus the sensitivity of macrophage to rigidity would promote a more specific ingestion of these pathogens. Furthermore, at sites of wounding and pathogenic invasion, an elevation of LPA levels (Moolenaar, 1999) would reduce the mechanical dependence of Fc-mediated phagocytosis and lead to more aggressive attacks of invading microbials irrespective of their rigidity. Conversely, it is also possible that improper regulation of mechanical sensing can lead to autoimmune diseases such as rheumatoid arthritis. Given the stiffness of cartilage relative to other tissues (Hayes and Mockros, 1971), its mechanical properties may play an important role during the progression of this disease. Defining mechanosensing in phagocytosis could facilitate the development of new clinical approaches that target the mechanosensing pathways of the cell to either promote the recognition of pathogens or interfere with autoimmune responses.

The authors thank Paul Janmey for sharing the procedure of EDC activation, Elizabeth Luna for critical reading of the manuscript, and $\mathrm{S}$. Narumiya for providing the $\mathrm{C} 3$ toxin. This study was supported by NIH NRSA grant GM-20578 to K.A.B., and NIH grant GM-32476 and NASA grant NAG2-1197 to Y.-L.W.

\section{References}

Aderem, A. and Underhill, D. M. (1999). Mechanisms of phagocytosis in macrophages. Annu. Rev. Immunol. 17, 593-623.

Allen, L. A. and Aderem, A. (1996). Molecular definition of distinct cytoskeletal structures involved in complement- and Fc receptor-mediated phagocytosis in macrophages. J. Exp. Med. 184, 627-637.

Allen, W. E., Jones, G. E., Pollard, J. W. and Ridley, A. J. (1997). Rho, rac and cdc42 regulate actin organization and cell adhesion in macrophages. $J$. Cell Sci. 110, 707-720.

Amano, M., Ito, M., Kimura, K., Fukata, Y., Chihara, K., Nakano, T., Matsuura, Y. and Kaibuchi, K. (1996). Phosphorylation and activation of myosin by rho-associated kinase (Rho-kinase). J. Biol. Chem. 271, 2024620249.

Botelho, R. J., Teruel, M., Dierckman, R., Anderson, R., Wells, A., York, J. D., Meyer, T. and Grinstein, S. (2000). Localized biphasic changes in phosphatidylinositol-4,5 bisphosphate at sites of phagocytosis. J. Cell Biol. 151, 1353-1368.

Bray, D. (1984). Axonal growth in respose to experimentally applied tension. Dev. Biol. 102, 379-389.

Candau, F. and Leong, Y. S. (1985). Kinetic studies of the polymerization of acrylamide in inverse microemulsion. J. Polymer Sci. 23, 193-214.

Cannon, G. J. and Swanson, J. A. (1992). The macrophage capacity for phagocytosis. J. Cell Sci. 101, 907-913.

Caron, E. and Hall, A. (1998). Identification of two distinct mechanisms of phagocytosis controlled by different rho GTPases. Science 282, 1717-1721.

Castellano, F., Montcourrier, P. and Chavier, P., (2000). Membrane recruitment of rac1 triggers phagocytosis. J. Cell Sci. 113, 2955-2961.

Chimini, G. and Chavier, P. (2000). Function of rho family proteins in actin dynamics during phagocytosis and engulfment. Nat. Cell Biol. 2, E191E196.

Chrzanowska-Wodnicka, M. and Burridge, K. (1996). Rho-stimulated contractility drives the formation of stress fibers and focal adhesions. J. Cell Biol. 133, 1403-1415.

Cox, D., Chang, P., Zhang, Q., Reddy, P. G., Bokoch, G. M. and Greenberg, S. (1997). Requirements for both rac 1 and cdc 42 in membrane ruffling and phagocytosis in leukocytes. J. Exp. Med. 186, 1487-1494.
Dharmawardhane, S., Brownson, D., Lennartz, M. and Bokoch, C. M. (1999). Localization of p21 activated kinase one (pak1) to pseudopodia, membrane ruffles, and phagocytic cups in activated human neutrophils. $J$. Leukocyte Biol. 66, 521-527.

Donaldson, K., Li, X. Y., Dogra, S., Miller, B. G. and Brown, G. M. (1992), Asbestos-stimulated tumour necrosis factor release from alveolar macrophages depends on fibre length and opsonization. J. Pathol. 168, 243248.

Doyle, R. J. and Marquis, R. E. (1994). Elastic, flexible peptidoglycan and bacterial cell wall properties. Trends Microbiol. 2, 57-60.

Greenberg, S. and Silverstein, S. C. (1993). In Fundamental Immunology. New York: Raven Press.

Greenberg, S., Chang, P. and Silverstein, S. C. (1993). Tyrosine phosphorylation is required for fc receptor-mediated phagocytosis in mouse macrophages. J. Exp. Med. 177, 529-534.

Greenberg, S., Chang, P. and Silverstein, S. C. (1994). Tyrosine phosphorylation of the gamma subunit of Fc gamma receptors, p72syk, and paxillin during $\mathrm{Fc}$ receptor-mediated phagocytosis in macrophages. J. Biol. Chem. 269, 3897-3902.

Hackam, D. J., Rotstein, O. D., Schreiber, A., Zhang, W.-J. and Grinstein, S. (1997). Rho is required for the initiation of calcium signaling and phagocytosis by Fc $\gamma$ receptors in macrophages. J. Exp. Med. 186, 955-966.

Hayes, W. C. and Mockros, L. F. (1971). Viscoelastic properties of human articular cartilage. J. App. Physiol. 31, 562-568.

Heidemann, S. R., Kaech, S., Buxbaum, R. E. and Matus, A. (1999). Direct observation of the mechanical behaviors of the cytoskeleton in living fibroblasts. J. Cell Biol. 145, 109-122.

Henson, P. M. (1971). Interaction of cells with immune complexes: adherence, release of constituents, and tissue injury. J. Exp. Med.134, 114s$135 \mathrm{~s}$.

Koh, J. S., Lieberthal, W., Heydrick, S. and Levine, J. S. (1998). Lysophosphatidic acid is a major serum noncytokine survival factor for murine macrophages which acts via the phosphatidylinositol 3-kinase signaling pathway. J. Clin. Invest. 102, 716-727.

Koval, M., Prieter, K., Adles, C., Stahl, P. D. and Steinberg, T. H. (1998), Size of IgG-opsonized particles determines macrophage response during internalization. Exp. Cell Res. 242, 265-273.

Kunioka, Y. and Ando, T. (1996). Innocous labeling of the subfragment-2 region of skeletal muscle heavy meromyosin with a fluorescent polyacrylamide nanobead and visualization of individual heavy meromyosin molecules. J. Biochem. 119, 1024-1032.

Lo, C.-M., Wang, H.-B., Dembo, M. and Wang, Y.-L. (2000). Cell movement is guided by the rigidity of the substrate. Biophys. J. 79, 144152.

Massol, P., Montcourrier, P., Guillemot, J.-C. and Chavier, P. (1998). Fc receptor-mediated phagocytosis requires CDC42 and Rac1. EMBO J. 17, 6219-6229.

May, R. C. and Machesky, L. M. (2001). Phagocytosis and the actin cytoskeleton. J. Cell Sci. 114, 1061-1077.

May, R. C., Caron, E., Hall, A. and Machesky, L. M. (2000). Involvement of the arp2/3 complex in phagocytosis mediated by Fc $\gamma \mathrm{R}$ or CR3. Nat. Cell Biol. 2, 246-248.

Metchnikoff, E. (1968). Lectures on the Comparative Pathology of Inflammation. New York: Dover Publications.

Moolenaar, W. H. (1999). Bioactive lysophospholipids and their G proteincoupled receptors. Exp. Cell Res. 253, 230-238.

Nobes, C. D. and Hall, A. (1999). Rho GTPases control polarity, protrusion, and adhesion during cell movement. J. Cell Biol. 144, 12351244.

North, R. J. (1968). The additive effects on the spreading of guinea pig macrophages of exogenous ATP and a surface coated with antigen-antibody molecules. Exp. Cell Res. 54, 267-268.

O'Connell, C. B., Wheatley, S. P., Ahmed, S. and Wang, Y.-L. (1999). The small GTP-binding protein rho regulates cortical activities in cultured cells during division. J. Cell Biol. 144, 305-313.

Pelham, R. J, and Wang, Y.-L. (1997). Cell locomotion and focal adhesions are regulated by substrate flexibility. Proc. Natl. Acad. Sci. USA 94, 1366113665.

Rabinovitch, M. and DeStefano, M. J. (1973). Macrophage spreading in vitro. I. Inducers of spreading. Exp. Cell Res. 77, 323-334.

Ridley, A. J. and Hall, A. (1992). The small GTP-binding protein rho regulates the assembly of focal adhesions and actin stress fibres in response to growth factors. Cell 70, 389-399. 
856 Journal of Cell Science 115 (4)

Riveline, D., Zamir, E., Balaban, N. Q., Schwarz, U. S., Ishizaki, T., Narumiya, S., Kam, Z., Geiger, B. and Bershadsky, A. D. (2001). Focal contacts as mechanosensors: externally applied local mechanical force induces growth of focal contacts by an mDia1-dependent and ROCKindependent mechanism. J. Cell Biol. 153, 1175-1186.

Schmidt, C., Pommerenke, H., Durr, F., Nebe, B. and Rychly, J. (1998). Mechanical stressing of integrin receptors induces enhanced tyrosine phosphorylation of cytoskeletally anchored proteins. J. Biol. Chem. 273, 5081-5085.

Sheetz, M. P., Felsenfeld, D. P. and Galbraith, C. G. (1998). Cell migration: regulation of force on extracellular matrix-integrin complexes. Trends Cell Biol. 8, 51-54.
Swanson, J. A. (1989). Phorbol esters stimulate macropinocytosis and solute flow through macrophages. J. Cell Sci. 94, 135-142.

Thwaites, J. J. and Mendelson, N. H. (1991). Mechanical behavior of bacterial cell walls. Adv. Microbiol. Physiol. 32, 173-222.

Verkhovsky, A. B., Svitkina, T. M. and Borisy, G. G. (1999). Selfpolarization and directional motility of cytoplasm. Curr. Biol. 9, 11-20.

Wang, Y.-L. (1994). Microinjection of proteins into somatic cells: needle microinjection and scrape loading. In Cell Biology, a Laboratory Handbook. (ed. J. E. Celis), pp. 16-21. San Diego: Academic Press.

Wang, Y.-L. and Pelham, R. J. (1998). Preparation of a flexible porous polyacrylamide substrate for mechanical studies of cultured cells. Methods Enzymol. 298, 489-496. 